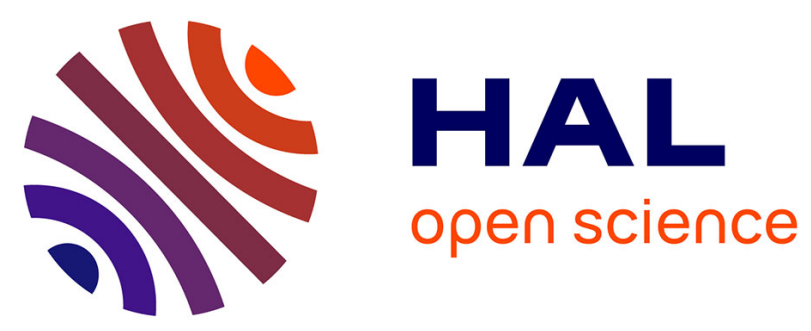

\title{
Modeling of Non-linear Relations among Different Design and Manufacturing Evaluation Measures for Multiobjective Optimal Concurrent Design
}

\author{
H. Yang, D. Xue, Y. L. Tu
}

\section{- To cite this version:}

H. Yang, D. Xue, Y. L. Tu. Modeling of Non-linear Relations among Different Design and Manufacturing Evaluation Measures for Multiobjective Optimal Concurrent Design. Concurrent Engineering: Research and Applications, 2006, 14 (1), pp.43-53. 10.1177/1063293X06063842 . hal-00571189

\author{
HAL Id: hal-00571189 \\ https://hal.science/hal-00571189
}

Submitted on 1 Mar 2011

HAL is a multi-disciplinary open access archive for the deposit and dissemination of scientific research documents, whether they are published or not. The documents may come from teaching and research institutions in France or abroad, or from public or private research centers.
L'archive ouverte pluridisciplinaire HAL, est destinée au dépôt et à la diffusion de documents scientifiques de niveau recherche, publiés ou non, émanant des établissements d'enseignement et de recherche français ou étrangers, des laboratoires publics ou privés. 


\title{
Modeling of Non-linear Relations among Different Design and Manufacturing Evaluation Measures for Multiobjective Optimal Concurrent Design
}

\author{
H. Yang, D. Xue* and Y. L. Tu \\ Department of Mechanical and Manufacturing Engineering, University of Calgary, \\ Calgary, Alberta, Canada T2N 1N4
}

\begin{abstract}
This research introduces a new approach to model the non-linear relations among different design and manufacturing evaluation measures for multiobjective optimal concurrent design. In this approach, different design and manufacturing evaluation measures are mapped to comparable evaluation indices. The non-linear relation between an evaluation measure and its evaluation index is identified based on the least-square curve-fitting method. The weighting factors for different design and manufacturing evaluation indices, representing the importance measures of these indices in the multiobjective design optimization, are achieved using the pair-wise comparison method. An example case study of automobile caliper disc brake design considering 3 design evaluation measures and 1 manufacturing evaluation measure is given to illustrate the effectiveness of the introduced approach.
\end{abstract}

Key Words: concurrent design, design performance, manufacturing cost, multiobjective optimization, least-square curve-fitting, pair-wise comparison method.

\section{Introduction}

\subsection{Multiobjective Optimal Concurrent Design}

Concurrent design is an approach to reduce product development lead time and improve the overall product life-cycle quality by integrating the design and other product development life-cycle aspects into the same environment [1,2]. In concurrent design, one or several downstream product development life-cycle aspects, such as manufacturing [3,4], assembly [5], maintenance [6,7], and disposal/recycle [8], are considered at early design stages.

Many methodologies and computer tools have been developed to improve the efficiency of concurrent design. Among these methodologies and tools, the optimization approach is effective to achieve the optimal design parameters when certain design evaluation objectives, such as design performance, manufacturing cost, maintenance cost, etc., are provided.

In most of the presently developed optimizationbased concurrent design methods, only one of the downstream product development life-cycle

*Author to whom correspondence should be addressed.

E-mail: dxue@ucalgary.ca

Figure 3 appears in color online: http://cer.sagepub.com evaluation aspects is considered. When several lifecycle aspects are considered, trade-off among these evaluation measures has to be conducted. Multiobjective optimization approach is effective for identifying the optimal design when several life-cycle evaluation measures, usually in different units, are considered [9].

Suppose the design parameter variables are described by a vector $\mathbf{X}=\left(x_{1}, x_{2}, \ldots, x_{n}\right)$, a multiobjective design optimization problem is formulated as

$$
\operatorname{Min} F(\mathbf{X})=\left\{f_{1}(\mathbf{X}), f_{2}(\mathbf{X}), \ldots f_{m}(\mathbf{X})\right\}
$$

subject to:

regional constraints; $\mathbf{X}_{L} \leq \mathbf{X} \leq \mathbf{X}_{U}$

inequality constraints: $G_{j}(\mathbf{X}) \leq 0, j=1,2, \ldots, p$

equality constraints: $H_{k}(\mathbf{X})=0, k=1,2, \ldots, q$ where $f_{i}(\mathbf{X}), i=1,2, \ldots, m$, is an objective function.

Presently many methods have been developed to solve multiobjective optimization problems [9]. These methods are primarily classified into three categories [10]. The methods in the first category convert a multiobjective optimization problem into a singleobjective optimization problem by assigning weights, preferences, utilities, or targets to the different objective functions [11]. The methods in the second category are used to first identify the multiple Pareto set points (i.e., the optimal solutions) and then allow the decision makers to select 
one based upon their selection criteria [12]. The methods in the third category try to model each single objective function and then explore the Pareto optimal frontier by using surrogate models or directly approximating the Pareto optimal functions [13]. Multiobjective optimization approach has also been widely employed in different types of engineering designs such as decision-based design $[14,15]$ and different types of engineering applications such as fuel cell application [10]. The research discussed in this article belongs to the first category of the multiobjective optimization methods.

\subsection{Problems in Modeling the Relations among Different Life-cycle Evaluation Measures}

Despite the progress, the following two problems need to be addressed for identifying the optimal design considering various life-cycle evaluation measures through multiobjective optimization.

\subsubsection{THE NON-LINEAR RELATIONS AMONG DIFFERENT LIFE-CYCLE EVALUATION MEASURES ARE NOT WELL MODELED}

The different life-cycle evaluation measures, such as power output of an engine, energy efficiency of the engine, and manufacturing cost of the engine, are usually modeled in different units. To compare these different evaluation measures, these measures are usually scaled into indices between 0 and 1 , such as the example shown in Figure 1. These evaluation indices usually do not provide any semantic meanings. In addition, the relation between the evaluation measure and the evaluation index is defined by a linear relation:

$$
I_{i}(\mathbf{X})=\alpha_{i} f_{i}(\mathbf{X})+\beta_{i}, \quad i=1,2, \ldots, m
$$

where $f_{i}(\mathbf{X})$ and $I_{i}(\mathbf{X})$ are the evaluation measure and the evaluation index, respectively, and $\alpha_{i}$ and $\beta_{i}$ are two coefficients. In engineering design, the relation between a life-cycle evaluation measure and its life-cycle evaluation index is not always a linear one. For example, customers are usually extremely satisfied when the stopping time of a car is within $1 \mathrm{~s}$. When the stopping time changes to $2 \mathrm{~s}$, the satisfaction measure decreases dramatically.

\subsubsection{THE WEIGHTING FACTORS FOR DIFFERENT LIFE-CYCLE EVALUATION MEASURES ARE USUALLY ASSIGNED IN AN AD HOC MANNER}

A multiobjective optimization problem can be converted into a singleobjective optimization problem by assigning weighting factors to different objective functions. Suppose $f_{1}(\mathbf{X}), f_{2}(\mathbf{X}), \ldots, f_{m}(\mathbf{X})$ are comparable objective functions, the objective function of this multiobjective optimization problem is then defined by:

$$
\operatorname{Max} F(\mathbf{X})=W_{1} f_{1}(\mathbf{X})+W_{2} f_{2}(\mathbf{X})+\ldots+W_{m} f_{m}(\mathbf{X})
$$

where $W_{1}, W_{2}, \ldots, W_{m}$ are weighting factors representing the importance of these evaluation measures. The weighting factors in Equation (3) are usually assigned base upon the experience of engineers. When a large number of life-cycle evaluation measures are considered, since most of the weighting factors are achieved by comparing with a reference weighting factor, inconsistency among weighting factors of these life-cycle evaluation measures sometimes occurs.

In the previous research of the authors, a method to obtain the optimal design considering functional performance and production cost was introduced $[16,17]$. In this method, functional performance and production cost are scaled into comparable functional performance index and production cost index, represented by values between 0 and 1. A systematic

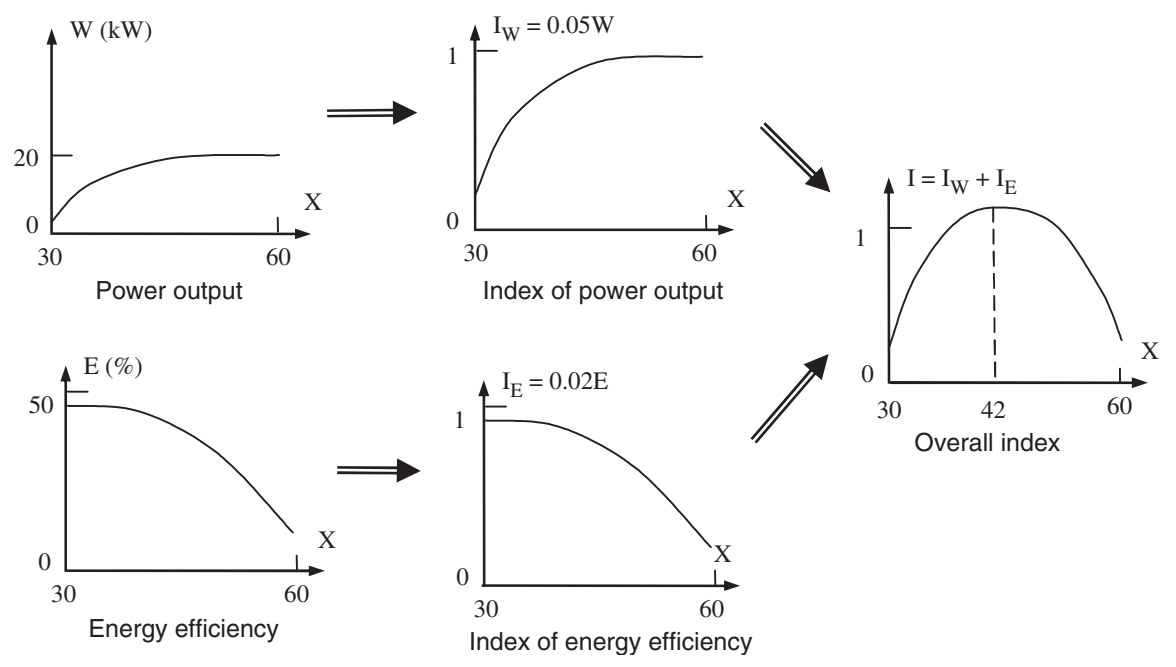

Figure 1. Linear relations between evaluation measures and evaluation indices. 
approach was introduced to achieve the design performance index and the production cost index by obtaining the increase (or decrease) measures of the functional performance and the production cost compared with a pre-selected reference design [18]. The introduced multiobjective optimization approach was also employed in the design of fuel cells, fuel cell stacks, and fuel cell systems [19].

The research presented in this article is a continuation of authors' previous work on multi-objective optimization [16-19]. In this research, the non-linear relations between life-cycle evaluation measures and their lifecycle evaluation indices are modeled using the leastsquare curve-fitting method [20]. The weighting factors of different life-cycle evaluation indices are identified using the pair-wise comparison method [21].

\section{Modeling of Non-linear Relations among Different Life-cycle Evaluation Measures}

Consider the multiobjective design optimization problem defined in Equation (1), suppose each lifecycle evaluation measure, $f_{i}(\mathbf{X})(i=1,2, \ldots, m)$, is converted to a life-cycle evaluation index, $I_{i}(\mathbf{X})$, using

$$
I_{i}(\mathbf{X})=F_{i}\left[f_{i}(\mathbf{X})\right], \quad i=1,2, \ldots, m
$$

where $I_{i}(\mathbf{X})$ is described by a number between 0 and 1 without unit, representing how good the design is considering the selected life-cycle evaluation measure, the multiobjective design optimization problem can then be modeled as

$$
\operatorname{Max} I(\mathbf{X})=W_{1} I_{1}(\mathbf{X})+W_{2} I_{2}(\mathbf{X})+\ldots+W_{m} I_{m}(\mathbf{X})
$$

where $W_{1}, W_{2}, \ldots, W_{m}$ are $m$ weighting factors for the $m$ life-cycle evaluation indices.

This research addresses the issues to identify the nonlinear relations, $F_{i}$, between the life-cycle evaluation measures, $f_{i}(\mathbf{X})$, and their life-cycle evaluation indices, $I_{i}(\mathbf{X})$, and to achieve the weighting factors, $W_{i}$, of these different life-cycle evaluation indices in a systematic approach.

\subsection{Modeling of Non-linear Relations between Life-cycle Evaluation Measures and Life-cycle Evaluation Indices Using the Least-square Curve-fitting Method}

The different life-cycle evaluation measures, $f_{i}(\mathbf{X})$, usually described in different units need to be converted into comparable life-cycle evaluation indices, $I_{i}(\mathbf{X})$, for identifying the optimal design using the multiobjective optimization approach. Each life-cycle evaluation index is described by a value between 0 and 1 , representing how good the design is, considering this particular lifecycle evaluation measure.

In the conventional multiobjective design optimization methods, different objective functions are compared directly, usually by scaling the objective functions into comparable measures (see example shown in Figure 1). In concurrent design, relations between lifecycle evaluation measures and life-cycle evaluation indices are usually non-linear relations. For example, Table 1 shows the relation between the stopping time of a car and its evaluation index representing the satisfaction of customers. The customers are extremely satisfied when the stopping time of a car is within $1 \mathrm{~s}$. When the stopping time changes to $2 \mathrm{~s}$, the satisfaction measure decreases dramatically due to the traffic rules.

In Table 1, the non-linear relation is defined by 4 pairs of discrete points. In optimization, however, a continuous function between a life-cycle evaluation measure and its life-cycle evaluation index is usually required to achieve the optimal design variable values. In this research, the least-square curve-fitting method [20] is used to identify this continuous function.

The non-linear relation between a life-cycle evaluation measure, $f_{i}(\mathbf{X})$, and its life-cycle evaluation index, $I_{i}(\mathbf{X})$, is defined as $p$-order polynomial in the form of

$$
\begin{aligned}
I_{i}(\mathbf{X})= & a_{0, i}+a_{1, i} f_{i}(\mathbf{X})+a_{2, i} f_{i}^{2}(\mathbf{X})+\ldots \\
& +a_{p, 1} f_{i}^{p}(\mathbf{X}), i=1,2, \ldots, m
\end{aligned}
$$

where, $a_{0, i}, a_{1, i}, \ldots, a_{p, i}$, are coefficients of the polynomial function. When a number of $f_{i}(\mathbf{X})$ values and their corresponding $I_{i}(\mathbf{X})$ values are given, the coefficients of the polynomial function, $a_{0, i}, a_{1, i}, \ldots, a_{p, i}$, in Equation (6) can be obtained using the least-square curve-fitting method.

Suppose $N$ values are obtained from $f_{i}(\mathbf{X})$ by changing the design variables in $\mathbf{X}$, and these values are described as $f_{1, i}, f_{2, i}, \ldots, f_{N, i},(i=1,2, \ldots, m)$, the corresponding life-cycle evaluation indices are described as $I_{1, i}$, $I_{2, i}, \ldots, I_{N, i}$, as shown in Figure 2, the $p+1$ coefficients, $a_{0, i}, a_{1, i}, \ldots, a_{p, i}$, of the polynomial function in

Table 1. Non-linear relation between car stopping time and customer satisfaction index.

\begin{tabular}{lc}
\hline : Car stopping time & $\begin{array}{c}\boldsymbol{I}_{\mathrm{T}} \text { : Customer } \\
\text { satisfaction index }\end{array}$ \\
\hline $0.5 \mathrm{~S}$ & 1.0 \\
$1.0 \mathrm{~S}$ & 0.8 \\
$1.5 \mathrm{~S}$ & 0.5 \\
$1.9 \mathrm{~S}$ & 0.1 \\
\hline
\end{tabular}


Equation (6) can be achieved by solving the following $p+1$ equations.

$$
\begin{aligned}
& a_{0, i} N+a_{1, i} \sum_{k=1}^{N} f_{k, i}+\ldots+a_{p, i} \sum_{k=1}^{N} f_{k, i}^{p} \\
& =\sum_{k=1}^{N} I_{k, i} \\
& a_{0, i} \sum_{k=1}^{N} f_{k, i}^{p}+a_{1, i} \sum_{k=1}^{N} f_{k, i}^{p+1}+\ldots+a_{p, i} \sum_{k=1}^{N} f_{k, i}^{2 p} \\
& \quad=\sum_{k=1}^{N} f_{k, i}^{p} I_{k, i} \quad i=1,2, \ldots, m
\end{aligned}
$$

where $N$ is the number of data points in Figure 2, $f_{k, i}$ is the $k$-th value for the $i$-th life-cycle evaluation measure, $I_{k, i}$ is the corresponding life-cycle evaluation index for the $f_{k, i}$, and $m$ is the total number of the life-cycle evaluation measures. Since the number of variables and the number of equations are the same for Equation (7), the unique coefficients, $a_{0, i}, a_{1, i}, \ldots, a_{p, i}$, can be calculated efficiently using the least-square curve-fitting method.

In this work, the cubic polynomial function $(p=3)$ is selected considering the quality and efficiency for solving the equations given in Equation (7).

\subsection{Identification of Weighting Factors for Different Life-cycle Evaluation Indices Using the Pair-wise Comparison Method}

The weights, $W_{i}$, in Equation (5) for different lifecycle evaluation measures, representing the importance factors of these evaluation measures, are usually assigned based on the experience of design engineers. When a large number of life-cycle evaluation measures are considered, since most of these weighting factors are achieved by comparing with a reference weighting factor, inconsistency among weighting factors of these life-cycle evaluation measures sometimes can be identified. In this research, the pair-wise comparison method [21] is employed to identify the weighting factors by comparing each of the life-cycle evaluation measures with all other life-cycle evaluation measures.

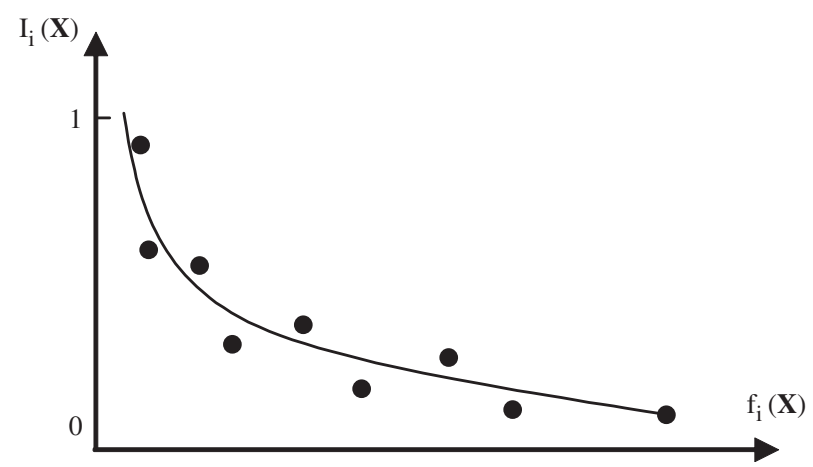

Figure 2. Least-square curve-fitting method.
The pair-wise comparison starts with comparing the relative importance, or importance ratio, of two selected items. If $m$ life-cycle evaluation indices are associated with $m$ weights, $W_{1}, W_{2}, \ldots, W_{m}$, the relative importance, $a_{i j}$, considering the $i$-th life-cycle evaluation index and the $j$-th life-cycle evaluation index is obtained as

$$
a_{i j}=\frac{W_{i}}{W_{j}} \quad i, j=1,2, \ldots, m
$$

The pair-wise ratios satisfy

$$
\left[\begin{array}{cccc}
a_{11} & a_{12} & \ldots & a_{1 m} \\
a_{21} & a_{22} & \ldots & a_{2 m} \\
\ldots & \ldots & & \\
a_{m 1} & a_{m 2} & \ldots & a_{m m}
\end{array}\right]\left[\begin{array}{c}
W_{1} \\
W_{2} \\
\ldots \\
W_{m}
\end{array}\right]=m\left[\begin{array}{c}
W_{1} \\
W_{2} \\
\ldots \\
W_{m}
\end{array}\right]
$$

Since a life-cycle evaluation index is equally important as itself (i.e., the value of a diagonal element in the matrix is 1), and the values of the elements in the upper triangle of the matrix are the reciprocal values of the elements in the lower triangle of this matrix, only $m(m-1) / 2$ times of comparisons are needed. Equation (9) can be simplified as

$$
A \mathbf{W}=m \mathbf{W}
$$

or

$$
(A-m I) \mathbf{W}=\mathbf{0}
$$

where $I$ is an $m \times m$ identity matrix. From this equation, it is apparent that $m$ is an eigenvalue of $A$, and $\mathbf{W}$ is an eigenvector for eigenvalue $m$.

If all the comparisons are perfectly consistent, the following relation should always be true for any combination of comparisons taken from the pair-wise comparison matrix $A$

$$
a_{i j}=a_{i k} a_{k j}, \quad i, j, k=1,2, \ldots, m
$$

where $m$ is the total number of the design evaluation indices.

However, for matrices created based on human judgment, the condition in Equation (12) does not hold all the time since human judgments are inconsistent to a greater or lesser degree. Therefore, perfect consistency rarely occurs in practice. In such a case, the vector $\mathbf{W}$ satisfies

$$
A \mathbf{W}=\lambda_{\max } \mathbf{W}
$$

where $\lambda_{\max }$ is the maximum eigenvalue of $A$ considering estimation errors. $\lambda_{\max }$ satisfies

$$
\lambda_{\max } \geq m
$$


The difference, if any, between $\lambda_{\max }$ and $m$ is an indication of the inconsistency of the judgments. The consistency index CI, introduced in [21], is the measure to evaluate the deviation from consistency of the pair-wise ratios. CI is calculated by

$$
\mathrm{CI}=\frac{\lambda_{\max }-m}{m-1}
$$

When values of the elements of a reciprocal matrix are generated randomly, the (CI) for this matrix is represented as random consistency index (RI). The average RI values for different orders of matrices are summarized in Table 2.

The ratio of CI to RI defined by

$$
\mathrm{CR}=\frac{\mathrm{CI}}{\mathrm{RI}}
$$

for the same order matrices is called the consistency ratio (CR). A pair-wise ratio matrix is considered to be adequately consistent, if the corresponding $\mathrm{CR}$ is less than 0.10 [21].

In the process to identify weighting factors of different life-cycle evaluation indices, designers are required to specify how a particular design evaluation index is more important than another index. The comparison values are selected on scales of 1-9 and their reciprocals, to form the $m \times m$ matrix $A$. The weighting factors of indices are obtained by identifying the maximum eigenvalue $\lambda_{\max }$ and the corresponding eigenvector, $\mathbf{W}=\left(W_{1}, W_{2}, \ldots, W_{m}\right)$, of the matrix $A$.

Table 2. Average consistency indices for random reciprocal matrices with different orders.

\begin{tabular}{ccccccccc}
\hline $\boldsymbol{m}$ & $\mathbf{2}$ & $\mathbf{3}$ & $\mathbf{4}$ & $\mathbf{5}$ & $\mathbf{6}$ & $\mathbf{7}$ & $\mathbf{8}$ & $\mathbf{9}$ \\
\hline $\mathrm{RI}$ & 0.00 & 0.58 & 0.90 & 1.12 & 1.24 & 1.32 & 1.41 & 1.45 \\
\hline
\end{tabular}

\section{A Case Study Example - Automobile Caliper Disc Brake Design}

A case study of automobile disc brake design is introduced in this section to demonstrate the effectiveness of the new multiobjective optimization based concurrent design approach by modeling the non-linear relations among the different life-cycle evaluation measures. In this case study, three design evaluation measures and one manufacturing evaluation measure are considered for achieving the optimal values of six design variables. This design example was developed based on the example provided in a textbook by Siddall [22].

\subsection{A Caliper Disc Brake Mechanism}

Most modern automobiles have disc brakes on the front wheels, and some have disc brakes on all four wheels. A brake system is used to stop the motion of the automobile. The single-piston floating caliper is often selected as the mechanism of the disc brake system. Figure 3(a) shows the configuration of the caliper disc brake system.

The high-pressure oil in the piston cylinder pushes the piston to apply forces to the brake pad linings to stop the motion of the rotor disc. The caliper is a selfadjusting mechanism and able to slide from side to side, so it can move to the center of the brake disc each time when the brake is applied.

Six design variables need to be obtained in this case study. These six design variables are given in Figure 3(b):

$D$ : outside disc diameter (in.)

$R$ : radius of center line of pad lining (in.)

$d$ : diameter of pad lining (in.)

$D_{\mathrm{p}}$ : diameter of piston (in.)

$a$ : thickness of disc (in.)

$p_{0}$ : oil pressure (psi)

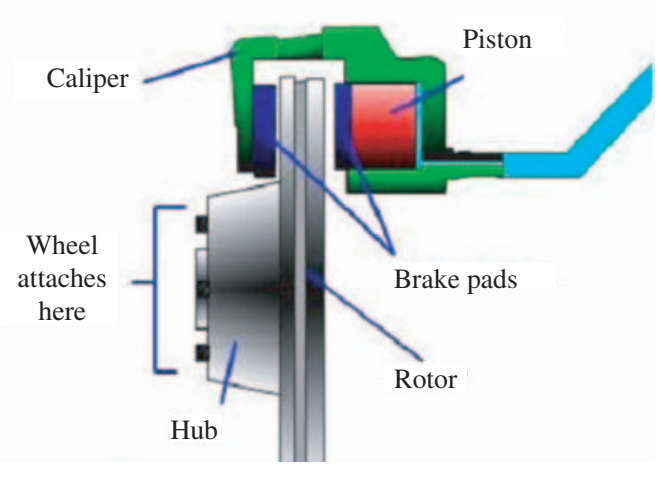

(a)

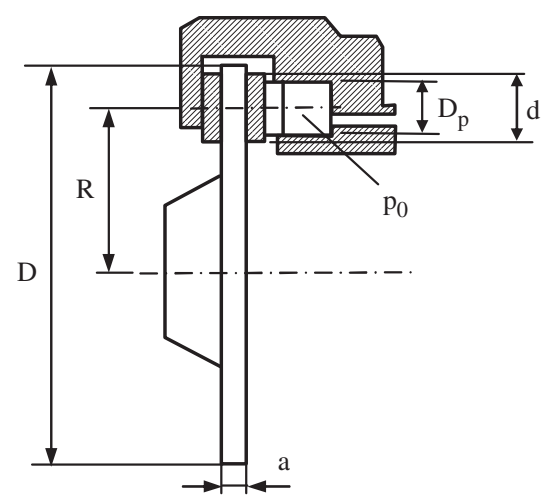

(b)

Figure 3. A caliper disc brake and its design variables. 


\subsection{Three Design Evaluation Measures and One Manufacturing Evaluation Measure}

Three design evaluation measures and one manufacturing evaluation measure are selected in this research to evaluate the caliper disc brake design: the stopping time, the final temperature of the disc, the pressure on the disc, and the manufacturing cost. The relations between the six design variables and the four life-cycle evaluation measures are formulated as follows.

\subsubsection{STOPPING TIME}

The stopping time is the time to stop the rotational motion of the brake disc by pressing the brake pedal. The kinetic energy of the vehicle is converted to heat in the discs during the braking process. The stopping time, $T$, measured in seconds, is calculated by

$$
T=\frac{12 E}{2 \pi \mu F I_{2} N} \times 60
$$

where $E$ is the kinetic energy of the vehicle measured in $\mathrm{ft} l \mathrm{~b}, \mu$ is the coefficient of friction (selected as 1 ), $F$ is the operating force of the pad on the disc measured in $1 b$, and $N$ is the initial rotational speed of the disc measured in rotation per minute $(\mathrm{rpm}) . I_{2}$ is calculated by

$$
I_{2}=4 \int_{R-d / 2}^{R+d / 2} \frac{r}{I_{1}} \tan ^{-1} \sqrt{\frac{(r+d / 2-R)(R+d / 2-r)}{(R+r+d / 2)(R+r-d / 2)}} \mathrm{d} r
$$

where $I_{1}$ is given by

$$
I_{1}=4 \int_{R-d / 2}^{R+d / 2} \tan ^{-1} \sqrt{\frac{(r+d / 2-R)(R+d / 2-r)}{(R+r+d / 2)(R+r-d / 2)}} \mathrm{d} r
$$

The kinetic energy, $E$, measured in $\mathrm{ft} \mathrm{lb}$ is given by

$$
E=\frac{1}{n} \frac{W V^{2}}{2 g}
$$

where $W$ is the vehicle weight (selected as $750 \mathrm{lb}$ ), $V$ is the vehicle initial velocity measured in $\mathrm{ft} / \mathrm{s}$ (selected as $50 \mathrm{mph}$, which is converted into $50 \times 1760 \times$ $3 / 3600=73.33 \mathrm{ft} / \mathrm{s}), g$ is the acceleration constant due to the gravity (selected as $32.15 \mathrm{ft} / \mathrm{s}^{2}$ ), and $n$ is the number of wheels (selected as 4).

The operating force of the pad on the disc, $F$, can be obtained by

$$
F=\frac{\pi D_{\mathrm{p}}^{2} p_{0}}{4}
$$

where $D_{\mathrm{p}}$ is the piston diameter measured in inches, $p_{0}$ is the oil pressure measured in psi.
The initial rotational speed of the disc, $N$, is given by

$$
N=\frac{V \times 1760 \times 3 \times 12}{2 \pi R_{t} 60}
$$

where $V$ is the vehicle initial velocity measured in $\mathrm{mph}$ (selected as $50 \mathrm{mph}$ ), $R_{t}$ is the radius of the tire (selected as 14 in.).

\subsubsection{FINAL TEMPERATURE OF DISC}

We assume that all energy is converted to heat in the disc. The final temperature of the disc must be lower than the maximum allowable temperature (selected as $500^{\circ} \mathrm{F}$ ). The final temperature of the disc, $t_{f}$, measured in ${ }^{\circ} \mathrm{F}$ is calculated by

$$
t_{f}=\frac{4}{778.3} \frac{E}{\pi c \rho D^{2} a}+t_{i}
$$

where $t_{i}$ is the initial or the ambient temperature measured in ${ }^{\circ} \mathrm{F}$ (selected as $75^{\circ} \mathrm{F}$ ), $c$ is the specific heat of disc measured in $\mathrm{Btu} / \mathrm{lb}{ }^{\circ} \mathrm{F}$ ( 0.12 for steel or cast iron), $\rho$ is the mass density of the disc measured in $1 \mathrm{~b} / \mathrm{in}^{3}{ }^{3}$ (0.2836 for steel), $E$ is the kinetic energy of the vehicle measured in $\mathrm{ft} \mathrm{lb}$, which is calculated using Equation (20), $D$ is the outside disc diameter measured in inches, and $a$ is the thickness of the disc measured in inches.

\subsubsection{PRESSURE ON DISC}

The amount of wear for the brake disc depends on the lining material, the pressure, and the number of operating cycles. In practice, this problem is handled in an empirical fashion by specifying a maximum allowable pressure for a given material, a given life, and a given application based on experiments. The pressure on disc, $p$, measured in psi is calculated by

$$
p=\frac{\pi D_{\mathrm{p}}^{2} p_{0}}{4 I_{1}(R-d / 2)}
$$

where $I_{1}$ is obtained using Equation (19), $D_{\mathrm{p}}$ is the diameter of piston measured in inches, $p_{0}$ is the oil pressure measured in psi, $R$ is the radius of center line of pad lining measured in inches, and $d$ is the diameter of pad lining measured in inches. The pressure of disc, $p$, must not exceed the maximum allowable pressure.

\subsubsection{MANUFACTURING COST}

The manufacturing cost is selected to evaluate the design from the manufacturing perspective. In this work, the material cost of the brake disc, the machining cost to produce the two surfaces of the brake disc, and 
the material cost of the two pad linings are considered. The total cost is calculated by

$$
C=c_{1} \frac{\pi D^{2}}{4} a+2 c_{2} \frac{\pi D^{2}}{4}+2 c_{3} \frac{\pi d^{2}}{4} h
$$

where

$c_{1}$; unit cost of disc material (selected as $\$ 0.2 /$ in. $^{3}$ )

$c_{2}$; unit cost of disc surface milling (selected as $\$ 0.16 /$ in. $^{2}$ ) in. ${ }^{3}$ )

$c_{3}$; unit cost of pad lining material (selected as $\$ 0.2 /$

$D$ : outside disc diameter (in.)

$a$ : thickness of disc (in.)

$d$ : diameter of pad lining (in.)

$h$ : thickness of pad lining (selected as $0.5 \mathrm{in}$.).

\subsection{Design Constraints}

Nine design constraints are considered for identifying the optimal design variable values based on the configuration of the caliper disc brake mechanism shown in Figure 3. These constraints are summarized in Table 3.

The eight constants used for defining the design constraints are selected as:

$D_{\mathrm{u}}$ : Maximum disc diameter (12 in.)

$D_{\mathrm{h}}$ : Hub diameter (3 in.)

$T_{\mathrm{u}}$ : Maximum allowable disc temperature $\left(500^{\circ} \mathrm{F}\right)$

$p_{\mathrm{u}}$ : Maximum allowable disc pressure (1500 psi)

$p_{\mathrm{m}}$ : Maximum available oil pressure (1000 psi)

$t_{\mathrm{c}}$ : Thickness of the cylinder wall $(0.25 \mathrm{in}$.)

$a_{\mathrm{u}}$ : Maximum thickness of the disc (1 in.)

$T_{\mathrm{m}}$ : Maximum allowable stopping time $(2 \mathrm{~s})$.

\subsection{Modeling of the Non-linear Relations between the Life-cycle Evaluation Measures and Their Life-cycle Evaluation Indices}

The four selected life-cycle evaluation measures, including the stopping time, $T$, the final temperature of the disc, $t_{\mathrm{f}}$, the pressure on the disc, $p$, and the manufacturing cost, $C$, are mapped to the four life-cycle evaluation indices, $I_{1}, I_{2}, I_{3}$, and $I_{4}$ according to Equation (4). The selected values for each of the four life-cycle evaluation measures and their corresponding values of the life-cycle evaluation indices are summarized in Table 4.

The data given in Table 4 only provide the discrete relations between the four life-cycle evaluation measures and their life-cycle evaluation indices. Since the optimal design variables are achieved using the multiobjective optimization approach, modeling of the relations between the four life-cycle evaluation measures and their life-cycle evaluation indices using continuous functions are required. In this research, the continuous relation between a life-cycle evaluation measure and its life-cycle evaluation index is described by a cubic polynomial function:

$$
\begin{aligned}
I_{i}(\mathbf{X})= & a_{0, i}+a_{1, i} f_{i}(\mathbf{X})+a_{2, i} f_{i}^{2}(\mathbf{X}) \\
& +a_{3, i} f_{i}^{3}(\mathbf{X}), \quad i=1,2,3,4
\end{aligned}
$$

The least-square curve-fitting method is used to identify the 4 coefficients of each cubic polynomial function. The achieved four cubic polynomial functions, representing the four life-cycle evaluation indices, are given in Equations (27)-(30).

$$
\begin{aligned}
I_{1}= & 1.1734-0.4163 T+0.1885 T^{2}-0.1404 T^{3} \\
I_{2}= & 1.0600+0.9524 \times 10^{-4} t_{f}-0.8214 \\
& \times 10^{-5} t_{f}^{2}+0.8333 \times 10^{-8} t_{f}^{3} \\
I_{3}= & 1.1045-0.9788 \times 10^{-4} p-0.4095 \\
& \times 10^{-6} p^{2}-0.2100 \times 10^{-12} p^{3} \\
I_{4}= & 2.31-0.0597 C+0.8765 C^{2}-0.5 \times 10^{-5} C^{3}
\end{aligned}
$$

The selected discrete data and the continuous functions achieved using the least-square curve-fitting method are shown in Figure 4.

Table 3. Design constraints.

\begin{tabular}{lc}
\hline Constraint & Inequality \\
\hline The outside diameter of the disc must be smaller than the maximum allowable diameter & $D_{\mathrm{h}} \leq D \leq D_{\mathrm{u}}$ \\
$\left(D_{\mathrm{u}}\right)$ and greater than the diameter of the hub $\left(D_{\mathrm{h}}\right)$. & $D / 2-R-\mathrm{d} / 2 \geq 0$ \\
The lining must not overhang the disc. & $R-\mathrm{d} / 2-D_{\mathrm{h}} / 2 \geq 0$ \\
The lining must not interfere with the hub $\left(D_{\mathrm{h}}\right.$ : the diameter of the hub). & $R-D_{\mathrm{p}} / 2-t_{\mathrm{c}}-D_{\mathrm{h}} / 2 \geq 0$ \\
The cylinder must not interfere with the hub $\left(t_{\mathrm{c}}\right.$ : thickness of the cylinder wall). & $p_{\mathrm{o}} \leq \mathrm{p}_{\mathrm{m}}$ \\
The oil pressure must not exceed the maximum available oil pressure $\left(p_{\mathrm{m}}\right)$. & $t_{\mathrm{f}} \leq T_{\mathrm{u}}$ \\
The final temprature of the disc must not exceed its maximum allowable temperature $\left(T_{\mathrm{u}}\right)$. & $p^{\prime} p_{\mathrm{u}}$ \\
The pressure on the disc must not exceed the maximum allowable pressure $\left(p_{\mathrm{u}}\right)$. & $a \leq a_{\mathrm{u}}$ \\
The thickness of the disc must not exceed the maximum allowable thickness $\left(a_{\mathrm{u}}\right)$. & $T \leq T_{\mathrm{m}}$ \\
The stopping time must not exceed the maximum allowable stopping time $\left(T_{\mathrm{m}}\right)$. & \\
\hline
\end{tabular}




\subsection{Identification of the Weighting Factors for the Life-cycle Evaluation Indices Using the Pair-wise Comparison Method}

The multiobjective optimization problem is formulated as

$$
\operatorname{Max} I=W_{1} I_{1}+W_{2} I_{2}+W_{3} I_{3}+W_{4} I_{4}
$$

where $W_{1}, W_{2}, W_{3}$, and $W_{4}$ are the four weighting factors of the four life-cycle evaluation indices.

Table 4. Life-cycle evaluation measures and their indices.

(a) Stopping time $t$ and index for stopping time $I_{1}$

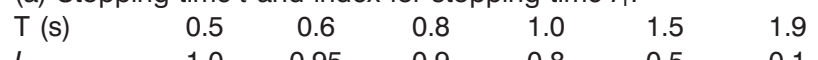

$\begin{array}{lllllll}1 & 1.0 & 0.95 & 0.9 & 0.8 & 0.5 & 0.1\end{array}$

(b) Final temperature $t_{f}$ and index for final temperature $I_{2}$.

$\begin{array}{llllll}t_{\mathrm{f}}\left({ }^{\circ} \mathrm{F}\right) & 100 & 200 & 300 & 400 & 500\end{array}$

$\begin{array}{llllll}I_{2} & 1.0 & 0.8 & 0.6 & 0.3 & 0.1\end{array}$

(c) Pressure $p$ and index for pressure $I_{3}$.

$\begin{array}{lllllll}p \text { (psi) } & 400 & 500 & 700 & 800 & 1200 & 1450 \\ l_{3} & 1.0 & 0.95 & 0.85 & 0.75 & 0.4 & 0.1\end{array}$

(d) Manufacturing cost $C$ and index for manufacturing cost $I_{4}$.

\begin{tabular}{llllll}
$C(\$)$ & 40 & 50 & 70 & 90 & 100 \\
\hline
\end{tabular}

$\begin{array}{llllll}I_{4} & 1.0 & 0.9 & 0.7 & 0.4 & 0.1\end{array}$
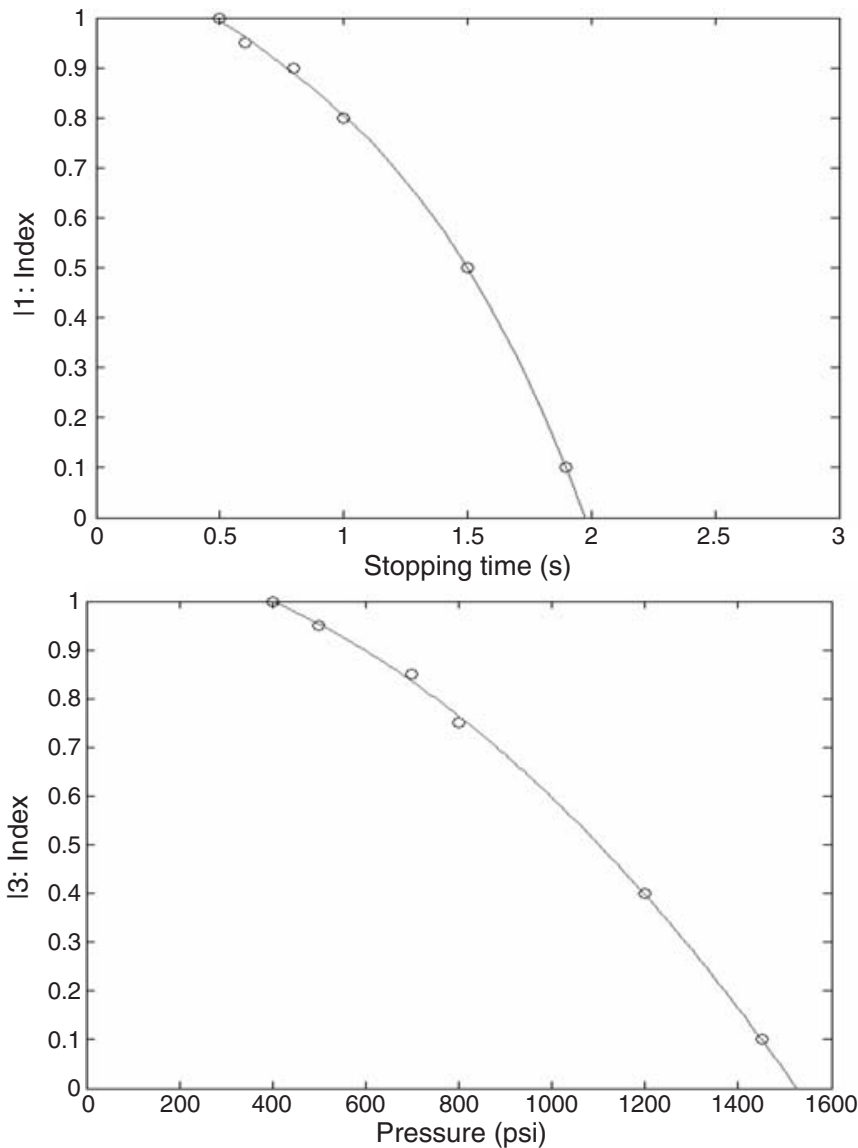

The pair-wise comparison method introduced in Section 2.2 is employed to achieve these weighting factors. Suppose the relative importance ratios for the four selected life-cycle evaluation indices are obtained as given in the Table 5, the equation to obtain the weighting factors is then defined as

$$
\left[\begin{array}{cccc}
1 & 7 & 5 & 3 \\
1 / 7 & 1 & 1 / 2 & 1 / 4 \\
1 / 5 & 2 & 1 & 1 / 6 \\
1 / 3 & 4 & 6 & 1
\end{array}\right]\left[\begin{array}{l}
W_{1} \\
W_{2} \\
W_{3} \\
W_{4}
\end{array}\right]=\lambda_{\max }\left[\begin{array}{l}
W_{1} \\
W_{2} \\
W_{3} \\
W_{4}
\end{array}\right]
$$

The maximum eigenvalue $\lambda_{\max }$ is obtained as 4.2094. The corresponding eigenvector is achieved as

$W=\left(W_{1}, W_{2}, W_{3}, W_{4}\right)=(0.551,0.062,0.088,0.299)$

From Equation (15), the CI, is obtained as

$$
\mathrm{CI}=\frac{\lambda_{\max }-m}{m-1}=\frac{4.2094-4}{4-1}=0.0698
$$

Since the comparison matrix $A$ is a fourth-order reciprocal matrix, the average RI, is selected as
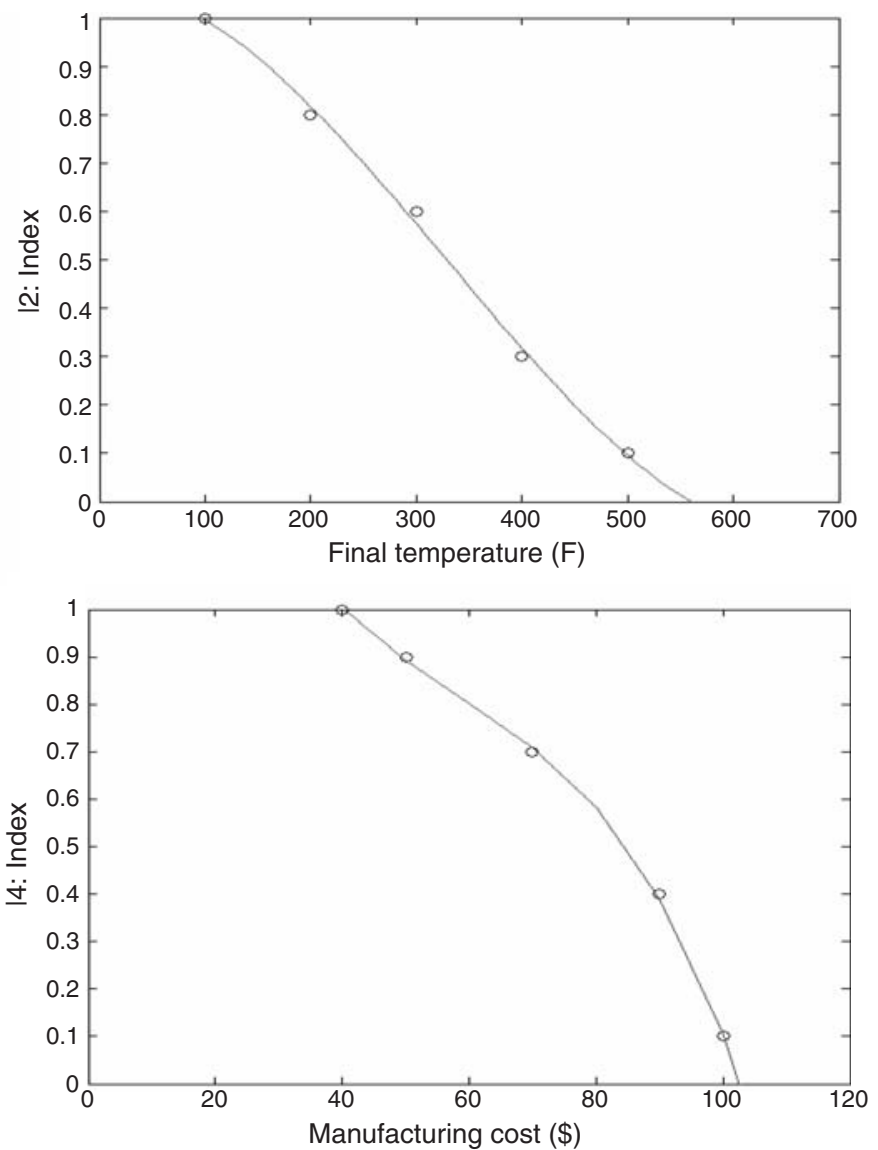

Figure 4. Continuous functions achieved using the least-square curve-fitting method. 
0.90 from Table 2. From Equation (16), the CR, is obtained as

$$
\mathrm{CR}=\frac{\mathrm{CI}}{\mathrm{RI}}=\frac{0.0698}{0.90}=0.0776
$$

Since the CR is less than 0.1 , the pair-wise ratio matrix is considered to be adequately consistent.

\subsection{Identification of the Optimal Design Variable Values}

The six design variables can be described as a vector

$$
X=\left(D, R, d, D_{\mathrm{p}}, a, p_{0}\right)
$$

The multiobjective optimization problem is converted into the following singleobjective optimization problem.

$$
\operatorname{Max} I(\mathbf{X})=0.551 I_{1}+0.062 I_{2}+0.088 I_{3}+0.299 I_{4}
$$

subject to:

$$
\begin{aligned}
& D_{\mathrm{h}} \leq D \leq D_{u} \\
& R+\frac{d}{2}-\frac{D}{2} \leq 0 \\
& \frac{d}{2}-R+\frac{D_{h}}{2} \leq 0 \\
& \frac{D_{\mathrm{p}}}{2}-R+t_{c}+\frac{D_{\mathrm{h}}}{2} \leq 0 \\
& t_{\mathrm{f}}-T_{h} \leq 0 \\
& p-p_{u} \leq 0 \\
& p_{0}-p_{m} \leq 0 \\
& a-a_{u} \leq 0 \\
& T-T_{m} \leq 0
\end{aligned}
$$

The optimal design variable values are obtained as

$$
X^{*}=(10.15,3.44,3.28,3.37,0.50,746.8)
$$

These optimal design variable values represent:

$D$ : outside disc diameter (10.15in.)

$R$ : radius of center line of pad lining (3.44 in.)

$d$ : diameter of pad lining (3.28 in.)

Table 5. Pare-wise comparison data.

\begin{tabular}{lcccc}
\hline & \multicolumn{4}{c}{ Second index } \\
\cline { 2 - 5 } First index & $\boldsymbol{I}_{\mathbf{1}}$ & $\boldsymbol{I}_{\mathbf{2}}$ & $\boldsymbol{I}_{\mathbf{3}}$ & $\boldsymbol{I}_{\mathbf{4}}$ \\
\hline$I_{1}$ & 1 & 7 & 5 & 3 \\
$I_{2}$ & $1 / 7$ & 1 & $1 / 2$ & $1 / 4$ \\
$I_{3}$ & $1 / 5$ & 2 & 1 & $1 / 6$ \\
$I_{4}$ & $1 / 3$ & 4 & 6 & 1 \\
\hline
\end{tabular}

$D_{\mathrm{p}}$ : diameter of piston $(3.37 \mathrm{in}$.)

$a$ : thickness of disc ( $0.50 \mathrm{in}$.)

$p_{0}$ : oil pressure (746.8 psi).

The four life-cycle evaluation measures corresponding to the optimal design are achieved as

$T=0.54 \mathrm{~s}$

$t_{\mathrm{f}}=133.6^{\circ} \mathrm{F}$

$p=793.3 \mathrm{psi}$

$C=61.6$ dollars

The overall life-cycle evaluation index is obtained as $I=0.9033$

\subsection{Discussion on the Optimal Design Result}

Section 3.6 demonstrates a case study to obtain the optimal design when all the four life-cycle evaluation measures are considered. When only some of these lifecycle evaluation measures are considered, different optimal designs are then achieved.

Table 6 shows the optimization results when only one of these life-cycle evaluation measure is considered. Compared with the optimal design created in Section 3.6, although the optimal design considering only one evaluation aspect provides the maximum evaluation measure in that aspect, this design is usually poorer in other evaluation aspects. The best evaluation measure for each of these optimal designs is described using bold font in Table 6 .

\section{Conclusions}

A new approach is introduced in this research to model the non-linear relations among different lifecycle evaluation measures for multiobjective optimal concurrent design. In this approach, different life-cycle evaluation measures are converted into comparable life-cycle evaluation indices. The least-square curvefitting method is employed to identify the non-linear relation between a life-cycle evaluation measure and its life-cycle evaluation index. The pair-wise comparison method is used to achieve the weighting factors for different life-cycle evaluation indices, representing the importance measures of these indices, in the multiobjective design optimization.

Characteristics of this research are summarized as follows.

1. By converting life-cycle evaluation measures into comparable life-cycle evaluation indices, concurrent design considering multiple different life-cycle evaluation measures in different units can be conducted.

2. By using the least-square curve-fitting method, the non-linear relations between life-cycle evaluation measures and life-cycle evaluation indices can be achieved. 
Table 6. The optimal designs considering different objective functions.

\begin{tabular}{|c|c|c|c|c|c|c|}
\hline Objective function & & $\operatorname{Max} I_{1}$ & $\operatorname{Max} I_{2}$ & $\operatorname{Max} I_{3}$ & $\operatorname{Max} I_{4}$ & Max I \\
\hline \multirow[t]{6}{*}{ The optimal design results } & $D$ & 11.36 in. & $11.99 \mathrm{in}$. & $8.55 \mathrm{in}$. & 8.22 in. & $10.15 \mathrm{in}$. \\
\hline & $R$ & $4.00 \mathrm{in}$. & 3.52 in. & $2.89 \mathrm{in.}$ & $2.96 \mathrm{in.}$ & $3.44 \mathrm{in}$. \\
\hline & $d$ & $3.26 \mathrm{in}$. & $1.38 \mathrm{in}$ & $2.77 \mathrm{in}$. & $2.30 \mathrm{in.}$ & $3.28 \mathrm{in.}$ \\
\hline & $D_{\mathrm{p}}$ & $3.70 \mathrm{in}$. & $1.82 \mathrm{in}$. & $2.27 \mathrm{in}$. & 2.43 in. & $3.37 \mathrm{in.}$ \\
\hline & $a$ & $1.00 \mathrm{in}$. & $1.00 \mathrm{in}$. & $1.00 \mathrm{in}$. & $0.50 \mathrm{in}$. & $0.50 \mathrm{in.}$ \\
\hline & $p_{0}$ & 707.3 psi & $655.2 \mathrm{psi}$ & $602.6 \mathrm{psi}$ & $777.8 \mathrm{psi}$ & $746.8 \mathrm{psi}$ \\
\hline \multirow[t]{4}{*}{ Design evaluation measures } & $T$ & $0.40 \mathrm{~s}$ & $2.00 \mathrm{~s}$ & $1.75 \mathrm{~s}$ & $1.15 \mathrm{~s}$ & $0.54 \mathrm{~s}$ \\
\hline & $t_{\mathrm{f}}$ & $98.4^{\circ} \mathrm{F}$ & $96.0^{\circ} \mathrm{F}$ & $116.3^{\circ} \mathrm{F}$ & $164.2^{\circ} \mathrm{F}$ & $133.6^{\circ} \mathrm{F}$ \\
\hline & $p$ & $829.5 \mathrm{psi}$ & $748.6 \mathrm{psi}$ & $84.5 \mathrm{psi}$ & $719.3 \mathrm{psi}$ & $793.3 \mathrm{psi}$ \\
\hline & $C$ & $\$ 86.8$ & $\$ 95.2$ & $\$ 49.4$ & $\$ 40.1$ & $\$ 61.6$ \\
\hline \multirow{5}{*}{ Design evaluation indices } & $l_{1}$ & 1.0000 & 0.0000 & 0.2686 & 0.7331 & 0.9817 \\
\hline & $I_{2}$ & 0.9978 & 1.0000 & 0.9731 & 0.8912 & 0.9461 \\
\hline & $I_{3}$ & 0.7414 & 0.8016 & 1.0000 & 0.8221 & 0.7690 \\
\hline & $I_{4}$ & 0.4620 & 0.2565 & 0.8968 & 1.0000 & 0.7896 \\
\hline & I & 0.8162 & 0.2090 & 0.5643 & 0.8305 & 0.9033 \\
\hline
\end{tabular}

3. By employing the pair-wise comparison method, a systematic approach to identify the weighting factors of different life-cycle evaluation indices is developed to keep the consistency of these weighting factors.

The effectiveness of the introduced approach has been demonstrated by a case study to obtain the six design variable values of an automobile caliper disc brake considering three design evaluation measures and one manufacturing evaluation measure.

\section{References}

1. Kusiak, A. (ed.) (1993). Concurrent Engineering: Automation, Tools, and Techniques, New York: John Wiley \& Sons.

2. Prasad, B. (1996). Concurrent Engineering Fundamentals: Volume I, NJ: Prentice Hall, Englewood Cliffs.

3. Bralla, J.G. (ed.) (1986). Handbook of Product Design for Manufacturing, New York, NY: McGraw-Hill.

4. Dong, Z. (1993). Design for Automated Manufacturing, In: Kusiak, A. (ed.), Concurrent Engineering: Automation, Tools, and Techniques, New York, NY: John Wiley \& Sons, pp. 207-234.

5. Boothroyd, G. and Dewhurst, P. (1983). Design for Assembly: A Designer's Handbook, Wakefield, RI: Boothroyd Dewhurst Inc.

6. Makino, A., Barkan, P. and Pfaff, R. (1989). Design for Serviceability, In: Proceedings of the 1989 ASME Winter Annual Meeting, San Francisco, CA.

7. Gershenson, J. and Ishii, K. (1993). Life-cycle Serviceability Design, In: Kusiak, A. (ed.), Concurrent Engineering: Automation, Tools, and Techniques, New York, NY: John Wiley \& Sons, pp. 363-384.

8. Zhang, H.C., Kuo, T.C., Lu, H. and Huang, S.H. (1997). Environmentally Conscious Design and Manufacturing: A State-of-the-Art Survey, Journal of Manufacturing Systems, 16(5): 352-371.

9. Roman, B., Statnikov, J. and Matusov, J.B. (1995). Multicriteria Optimization and Engineering, New York: Chapman and Hall.
10. Wang, G.G. and Shan, S. (2004). An Efficient Pareto Set Identification Approach for Multiobjective Optimization on Black-box Functions, In: Proceedings of The 2004 ASME International Design Engineering Technical Conferences and Computers and Information in Engineering Conference, Salt Lake City, Utah.

11. Ponnambalam, S.G., Jagannathan, H., Kataria, M. and Gadicherla, A. (2004). A TSP-GA Multi-objective Algorithm for Flow-shop Scheduling, International Journal of Advanced Manufacturing Technology, 23(11-12): 909-915.

12. Farmani, R., Savic, D.A. and Walters, G.A. (2005). Evolutionary Multi-objective Optimization in Water Distribution Network Design, Engineering Optimization, 37(2): 167-183.

13. Li, Y., Fadel, G.M. and Wiecek, M.M. (1998). Approximating Pareto Curves using the Hyper-ellipse, In: The 7-th AIAA/USAF/NASA/ISSMO Symposium on Multidisciplinary Analysis and Optimization, St. Louis, Missouri.

14. Mistree, F., Hughes, O.F. and Bras, B.A. (1993). The Compromise Decision Support Problem and the Adaptive Linear Programming Algorithm, Structural Optimization: Status and Promise, AIAA, Washington, DC, 247-289.

15. Chen, W., Wiecek, M.M. and Zhang, J. (1998). Quality Utility - A Compromise Programming Approach to Robust Design, In: Proceedings of the 1998 ASME International Design Engineering Technical Conferences and Computers and Information in Engineering Conference, Atlanta, Georgia.

16. Xue, D. and Dong, Z. (1993). Feature Modeling Incorporating Tolerance and Production Process for Concurrent Design, Concurrent Engineering: Research and Applications, 1(2): 107-116.

17. Xue, D. and Dong, Z. (1994). Developing a Quantitative Intelligent System for Implementing Concurrent Engineering Design, Journal of Intelligent Manufacturing, 5(4): 251-267.

18. Xue, D., Rousseau, J.H. and Dong, Z. (1996). Joint Optimization of Performance and Costs in Integrated Concurrent Design: Tolerance Synthesis Part, Engineering Design and Automation, 2(1): 73-89. 
19. Xue, D. and Dong, Z. (1998). Optimal Fuel Cell System Design Considering Functional Performance and Production Costs, Journal of Power Sources, 76(1): 69-80.

20. Hoffman, J.D. (1992). Numerical Methods for Engineers and Scientists, New York, NY: McGraw-Hill.

21. Saaty, T.L. (1980). The Analytic Hierarchy Process, New York, NY: McGraw-Hill.

22. Siddall, J.N. (1982). Optimal Engineering Design: Principles and Applications, New York, NY: Marcel Dekker Inc.

\section{H. Yang}

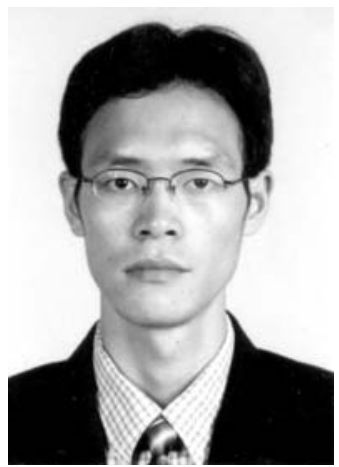

H. Yang is a PhD candidate at the Department of Mechanical and Manufacturing Engineering, University of Calgary. He received his $\mathrm{BSc}$ degree and MSc degree in Mechanical Engineering from University of Science and Technology of China in 1997 and 2000, respectively. His research interests include concurrent engineering, design modeling, and distributed system modeling.

\section{Xue}

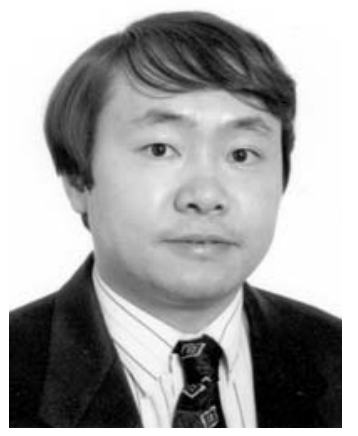

D. Xue is an Associate Professor at the Department of Mechanical and Manufacturing Engineering, University of Calgary. He received his $\mathrm{PhD}$ and MSc degrees in Precision Machinery Engineering from the University of Tokyo, and his BSc degree in Precision Instrument Engineering from Tianjin University. His research interests include product life-cycle modeling and integrated concurrent design, intelligent planning, scheduling, and control, design methodology and intelligent CAD, engineering optimization, tolerance modeling, engineering applications of artificial intelligence, and engineering applications of object oriented programming. He is a member of ASME, SME, AAAI, and IPSJ.

\section{Y. L. Tu}

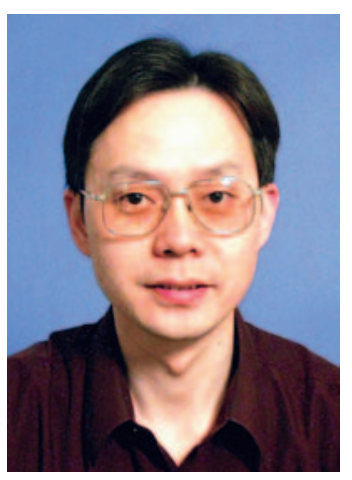

Y. L. Tu received his $\mathrm{BSc}$ degree in electronic engineering and his MSc in mechanical engineering both from the Huazhong University of Science and Technology (HUST), Peoples Republic of China, respectively in 1982 and 1985. From 1985 to 1990 , he was an Assistant Professor and Lecturer consecutively at the Department of Mechanical Engineering (1) of HUST. In 1993, he received his PhD from Aalborg University in Denmark and then worked at the Department of Production as a Postdoctoral research fellow from 1993 to 1995. From 1995 to 1997, he worked as an Assistant Professor at the Department of Manufacturing Engineering and Engineering Management, City University of Hong Kong. Between 1997 and 2003, he worked consecutively as a Lecturer and Senior Lecturer at the Department of Mechanical Engineering, University of Canterbury, New Zealand. Since 2002, he has been an Associate Professor at the Department of Mechanical and Manufacturing Engineering, University of Calgary, Canada. His present research interests are One-of-aKind Production (OKP) product design and manufacture and ultra-fast laser micro-machining. He has published dozens of research papers in international academic journals, two books and a number of book chapters. He is a senior member of SME (Society of Manufacture Engineers), member of IPENZ (Institution of Professional Engineers New Zealand) and a professional engineer of APEGGA (The Association of Professional Engineers, Geologists, and Geophysicists of Alberta). 